\title{
Frequency Modulated Continuous Wave System for Optical Fiber Intensity Sensors With Optical Amplification
}

\author{
Rosa Ana Perez-Herrera, Orlando Frazão, Jose Luis Santos, Francisco. M. Araújo, Luis A. Ferreira, \\ José M. Baptista, Member, IEEE, and Manuel Lopez-Amo, Senior Member, IEEE
}

\begin{abstract}
We report on the use of erbium doped fiber (EDF) amplification to enhance a frequency modulated continuous wave (FMCW) technique for referencing optical intensity sensors located between two Bragg grating structures. The experiment combines the concept of FMCW with the spectrally selective mirror properties of Bragg gratings to interrogate with referencing properties intensity based sensors. The interrogation system without amplification yields a sensor resolution of around $0.078 \mathrm{~dB}$. When the EDF amplifier is introduced into the experimental set up, the sensor sensitivity does not change, but the signal-to-noise ratio is improved, resulting into an enhanced resolution of $0.025 \mathrm{~dB}$. We also obtain a remote sensing operation at a location of $50 \mathrm{~km}$, showing the feasibility of this configuration to be used as a remote sensing application.
\end{abstract}

Index Terms-Fiber Bragg gratings (FBGs), frequency-modulated continuous wave, intensity sensors, optical amplification, selfreferenced sensors.

\section{INTRODUCTION}

$\mathbf{O}$ PTICAL fiber intensity-modulated sensors are very attractive since they are reliable, small-sized and suitable for a wide range of applications at lower costs [1]. However, to ensure accurate measurements, the implementation of a reference channel in the sensor is essential. Frequency-modulated continuous wave (FMCW) interference, which was originally investigated in electric radar, has been also successfully introduced in optics [2], [3]. The FMCW concept can be used to implement referentiation of intensity sensors. Optical FMCW interference has been demonstrated to have a number of attractive features, such as high resolution, large measurement range,

Manuscript received December 11, 2008; revised July 10, 2009; accepted July 17, 2009. This work was supported in part by the Spanish Comisión Interministerial de Ciencia y Tecnología within project TEC2007-67987-C02-02, in part by the European COST-299 action, and in part by FEDER funds. The associate editor coordinating the review of this paper and approving it for publication was Prof. Giorgio Sberveglieri.

R. A. Perez-Herrera and M. Lopez-Amo are with the Department of Electric and Electronic Engineering, Public University of Navarra, Pamplona, Spain (e-mail: rosa.perez@unavarra.es).

O. Frazão and J. L. Santos are with INESC Porto, Rua do Campo Alegre, 687, 4169-007 Porto, Portugal and also with the Department de Física, Faculdade de Ciências da Universidade do Porto, Rua do Campo Alegre, 687, 4169-007 Porto, Portugal.

F. M. Araújo and L. A. Ferreira are with INESC Porto, Rua do Campo Alegre, 687, 4169-007 Porto, Portugal.

J. M. Baptista is with INESC Porto, Rua do Campo Alegre, 687, 4169-007 Porto, Portugal and also with the Department de Matemáticas e Engenharias, Universidade da Madeira, 9300-390 Funchal, Portugal.

Digital Object Identifier 10.1109/JSEN.2009.2030391 and the capability of absolute measurement; it has also been widely used to construct various optical FMCW interferometers, fiber-optic FMCW interferometers, and fiber-optic FMCW interferometric sensors [4]. These developments deal with optical FMCW, i.e, it is the optical frequency that is chirped modulated in a particular format, but it is also feasible and often more simpler to perform subcarrier FMCW, where now it is the intensity of the light source that is chirped modulated. It has been demonstrated that this approach can also be effectively applied in fiber-optic sensing configurations [5].

Power budget has been shown to be a main limiting parameter in large scale multiplexing of intensity-based sensors or for remote sensing. The concept of remote sensing is applied to fiber-optic sensor systems ranging from $2 \mathrm{~m} \mathrm{[6]} \mathrm{up} \mathrm{to} 230 \mathrm{~km}$ [7] depending of the application. Most part of recently reported remote sensing systems consider $50 \mathrm{~km}$ as the reference length [8]-[11].

In order to overcome the limitation due to power budged, some networks employing different kinds of amplification have already been proposed and demonstrated [6], [13], [14]. Still, the inclusion of active fiber within the network originates a new source of noise, namely, amplified spontaneous emission noise (ASE), which reduces the optical signal-to-noise ratio (OSNR) of the system and, therefore, limits the operation range and sensitivity of the multiplexed sensors [1], [6]. Intensity-sensor networks using optical amplification open a broad range of sensing applications. Also, suppression of ASE effects in optically amplified sensor networks has been detected as a major issue and, therefore, it has been carried out the development of ASE immune techniques and topologies to interrogate fiber sensors [15], [16]. Work on referencing techniques for amplified systems can be found in [15], [17], and [18]. Such techniques should provide insensitivity to source intensity fluctuations, to variable optical transmission losses in the fiber link, or in couplers and connectors, all of them often indistinguishable from transducer caused effects.

In this work, the authors introduce optical gain to enhance a subcarrier FMCW technique based on fiber Bragg gratings (FBGs) for self-referenced interrogation of fiber-optic intensity sensors. In the first studied topology, an EDFA was inserted into the system in order to analyze the improvement of the sensor resolution without being affected by ASE. On the other hand, the second one includes also Raman amplification in order to 


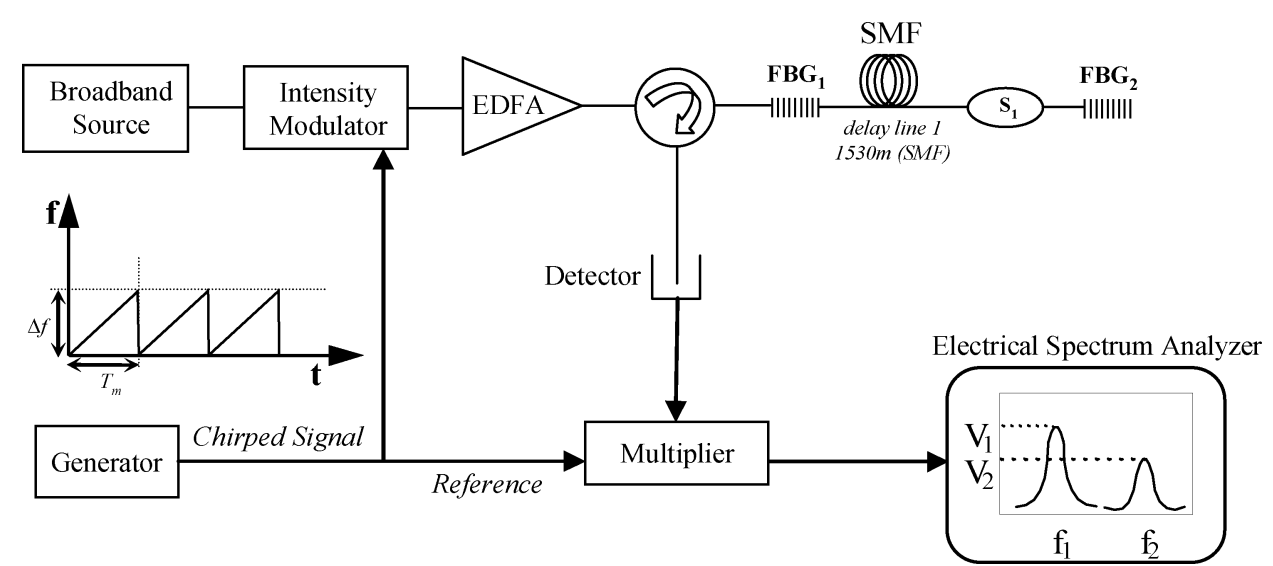

Fig. 1. Test setup for evaluation of the FMCW-based referentiation technique with optical gain by means of an EDFA.

demonstrate the possibility of remote sensing. For this last study, $50 \mathrm{~km}$ of SMF were inserted before the first FBG that conforms the sensing block.

\section{THEORY}

The basic concept of subcarrier optical FMCW interference is as follows [19], [20]: an optical wave, in which the optical power is sinewave modulated with a frequency that is linearly chirped following a triangular waveform that has frequency $f_{\text {mod }}$, is first divided into two waves. These two waves travel along different paths and then recombine at a point in space, originating a beat wave $f_{\text {beat }}$ with a frequency

$$
f_{\text {beat }}=2 \cdot \Delta f \cdot f_{\text {mod }} \cdot \tau=m \cdot \tau
$$

where the delay time $(\tau)$ is described as

$$
\tau=2 \cdot L \cdot \frac{n}{c}
$$

and $L$ is the length difference between the two paths, $\Delta f$ the amplitude of the frequency sweep which has a time rate $m$, and $c / n$ the wave speed ( $n$ is the medium refractive index). A detailed analysis reveals that the spectrum of the beat note consists of a group of discrete lines with $f_{\text {mod }}$ interval and a sinc-function envelope [21]. By choosing appropriate values of $\Delta f$ and $L$ in (1) and (2), the beat signal coincides with one of the harmonics of $f_{\text {mod }}$, i.e.,

$$
f_{\text {beat }}=k \cdot f_{\text {mod }}
$$

where $k$ is an integer. One advantage with the FMCW approach is the possibility to optimize the system for a certain application. With the FMCW, for a given value of $\Delta f$ one can choose a value of $f_{\text {mod }}$ that suits the application, as long as $1 / f_{\text {mod }}$ is larger than the optical round trip time. For instance, if the technique is used for distance determination, a larger value of $f_{\bmod }$ will improve the measurement resolution.

The FMCW concept can be used to implement referentiation of intensity sensors. As shown in Fig. 1, using FBG reflectors two signals are generated, one that will work as a reference and another one that has impressed the power modulation induced in the sensing head by the measurand. With proper parameter tuning, after detection the mixing of these two signals with the electronic chirped reference generates two beat signals at frequencies $f_{1}$ (with measurand information) and $f_{2}$, with amplitudes $V_{1}$ and $V_{2}$. The ratio of these two amplitudes,

$$
R \equiv \frac{V_{1}}{V_{2}}
$$

is defined as the measurement parameter, which is only dependent on the measurand induced loss. A preliminary validation of this concept has been previously reported by the authors [22]. Here, it is explored the effect on the system performance of having optical gain.

\section{NETWORK ANALYSIS}

For multiple sensor arrays the output signal spectrum will be a superposition of individual sensor signals in the frequency domain. If the sensor array is designed in such a way that each sensor signal aligns to a different harmonic of the frequency chirping, a maximum number sensor may be multiplexed with a determinate crosstalk between any two of the sensors. As is known, the nonzero sidelines would cause crosstalk between sensors.

It is interesting to note that the magnitude of the sidelines for the combined signal from more than one sensor can be actually smaller than that for one sensor, indicating that the spectral components of the sensors at a particular frequency $\left(k f_{s}\right)$ are not always added in phase.

The crosstalk between sensors due to nonzero sidelines could be negligible if the beat frequency from a FBG sensor coincides with one of the harmonics of the modulating frequency.

In a serial FBG array however, crosstalk may result from "multiple-reflection" and "spectral-shadowing" effects if the reflection spectra of two or more gratings are overlapping. Multiple reflection between the gratings will generate residual reflected waves, which will produce residual beat signals in the output frequency spectrum. Some of the waves that travel similar distances with the grating signal may have a significant effect on the system performance. This crosstalk exists for any serial connected sensor array, no matter what type of address 


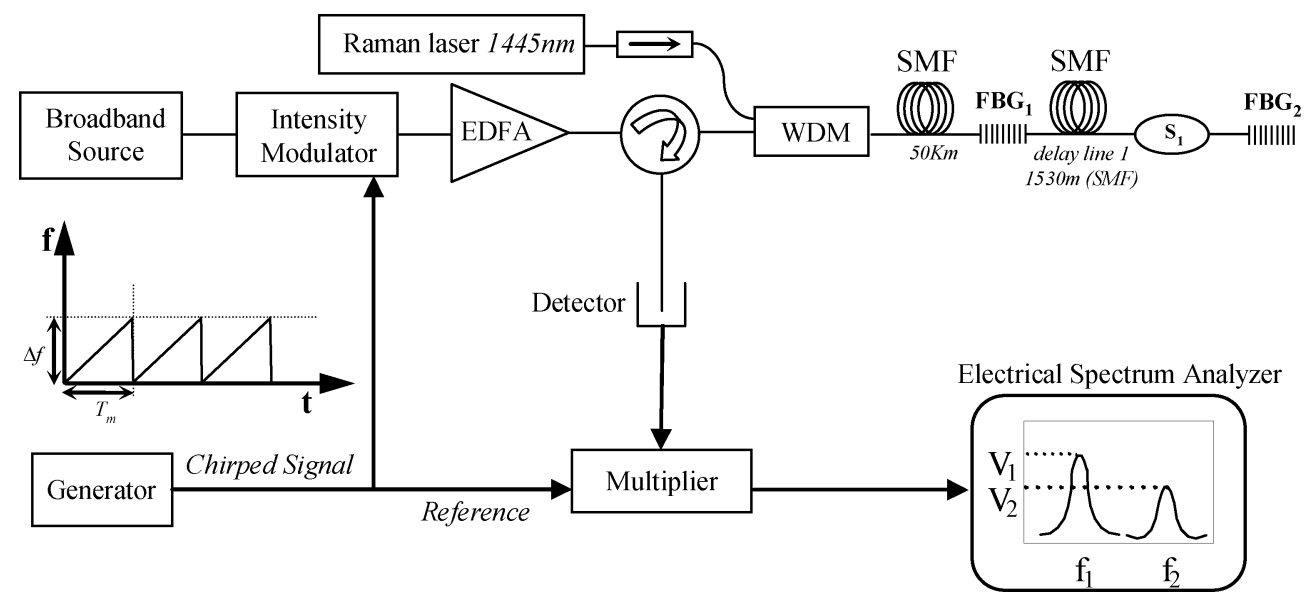

Fig. 2. Experimental setup incorporating Raman amplification for remote-sensing applications.

format (e.g., TDM, code-division multiple-access (CDMA), or FMCW) is used.

A detailed analysis of the effect of the multiple reflection on the crosstalk performance has been reported in [23]. The spectral shadowing crosstalk can be induced from upstream FBG sensors to downstream FBG sensors [24].

Both multiple reflection and spectral shadowing effects can be reduced by using low reflectivity gratings [23], [24]. However, the use of low reflectivity reduces the signal power of individual sensors at the output, thereby reducing the SNR. In order to maintain a reasonable power level at the photodetector, fiber amplifiers may need to be used before or after the sensor array to enhance the SNR [17]. Crosstalk from multiple reflection and spectral shadowing should not happen in a parallel topology system because the FBG sensors are located in different fiber branches, the light reflected from a particular sensor would not be influenced by other sensors.

\section{EXPERIMENTAL SETUP}

The first experiment was conducted using the setup shown in Fig. 1. It is a FMCW FBG array system, with the optical power enhanced by an EDFA. A broadband source based on an erbium doped fiber with an output power of $80 \mathrm{~mW}$ and bandwidth of $100 \mathrm{~nm}$ was used. The light from this source was modulated by an acousto-optic intensity modulator in which the chirped signal from the generator was applied. The frequency modulated signal provided from the generator was used as reference and applied to one of the multiplier inputs.

The experimental setup incorporated two FBGs, a delay line to separate the frequency response of each one, and an intensity sensing head. The intensity sensing head is composed by a tapered single-mode optical fiber with transmittance dependent on its curvature [25]. The wavelengths of FBGs are 1549.27 and $1549.93 \mathrm{~nm}$, having a reflectivity of $70 \%$ and a bandwidth of 0.2 $\mathrm{nm}$. An additional advantage of this structure is that the resonant wavelengths could have either identical or different values; however, there are advantages to consider different values because this increases the flexibility to choose the grating reflectivity for better operation.
Due to the modulation of the optical power injected into the system, the time delay between the reference signal and the modulated signal (which is enhanced using the delay line that can have a substantially reduced length if fast chirp rates are considered) is translated to a difference between the beat electric frequencies generated by multiplying the reflected signals with a local electronic reference. The amplitude of these beat frequencies was determined using an electric spectrum analyzer (ESA).

For these types of configurations, Fresnel reflected signals from the connectors are not an important problem due to the directivity of the couplers we used (about $-55 \mathrm{~dB}$ ). In addition to this, all the free terminations have been immersed in refractiveindex-matching gel to avoid undesired reflections. According our calculations, the return loss of an unprepared end is about 18 $\mathrm{dB}$ and commercial optical fiber connectors are available with return losses that exceed $50 \mathrm{~dB}$. For that reason, we can assure that using these commercial components, the reflected signals have hardly influence on the measurement we have taken.

To study the effect of optical amplification in the performance of the system, an EDFA, with $10 \mathrm{~dB}$ of gain and a bandwidth of $100 \mathrm{~nm}$, was located between the acousto-optic intensity modulator and the three ports circulator.

\section{REMOTE SENSING}

Within the sensor-application field there are several causes for including optical amplification: one of them is to enable the possibility of remote sensing. This motivates a second objective in our research: to demonstrate the performance of this structure for remote-sensing applications.

In order to enable remote operation at $50 \mathrm{~km}$, additional amplification has been introduced by means of a Raman pump laser. This Raman pump laser from IPG Fibertech radiates at $1445 \mathrm{~nm}$ and it could deliver up to $3 \mathrm{~W}$ power into a single-mode fiber. As can be seen in Fig. 2, the setup was modified by introducing the Raman pump laser by means of a WDM. In addition to this, $50 \mathrm{~km}$ of SMF were located before $\mathrm{FBG}_{1}$ in order to study the system behavior when the intensity sensor is located far away from the system header. Hybrid Raman-EDFA amplification systems has demonstrated optical signal-to-noise ratios higher than those of the discrete amplifier systems [26]. 


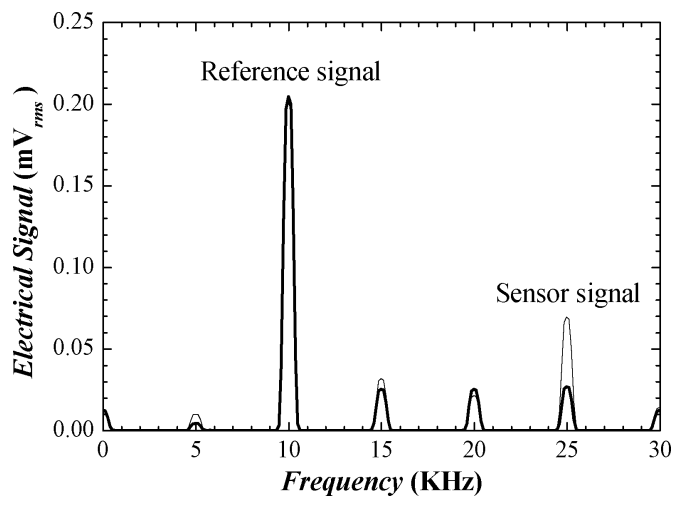

Fig. 3. Reference and signal spectral signatures in the presence of different losses in the sensing head.

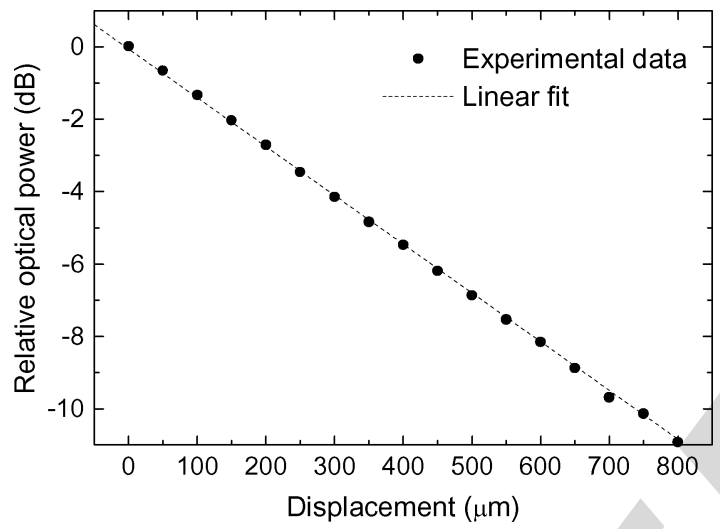

Fig. 4. Response of the fiber taper sensor.

\section{RESULTS AND DISCUSSIONS}

Computer simulations have been conducted to understand the effect of varying the parameters on the spectral characteristics of the beat note spectrum. All the parameters such as delay time, frequency deviation, and modulation frequency among others had been fine tuned before characterization of the system. In this case, the frequency sweep applied to the carrier followed a sawtooth modulation $\left[f_{\text {mod }}=5 \mathrm{kHz}, \Delta f=105 \mathrm{kHz}\right.$, $f_{0}$ (the sweep starting frequency) $=200 \mathrm{kHz}$. Fig. 3 shows the response of the beat note spectra of the optical intensity sensor based on Bragg grating. The first peak corresponds to the referencing signal and is related to the Bragg grating located before the sensing head, $\mathrm{FBG}_{1}$. The second peak corresponds to the signal of the optical intensity sensor and comes from $\mathrm{FBG}_{2}$. The frequency separation between these signatures is due to the fiber delay line located between the FBGs.

In this experiment, a low-cost optical intensity sensor based on a fused fiber taper was used. Fig. 4 shows an example of the response of this fiber taper sensor.

This structure was previously used by the authors to measure temperature, displacement, strain, and other parameters [25], [27], [28] and [29]. Its main feature is that when curvature is applied the insertion loss of the device changes, as can be also observed in Fig. 3.

Fig. 5 shows the plot of the R-parameter for the situations of amplification on and off. The pump power of the EDFA was adjusted to provide a signal amplified optical power of $\approx 8 \mathrm{~mW}$.

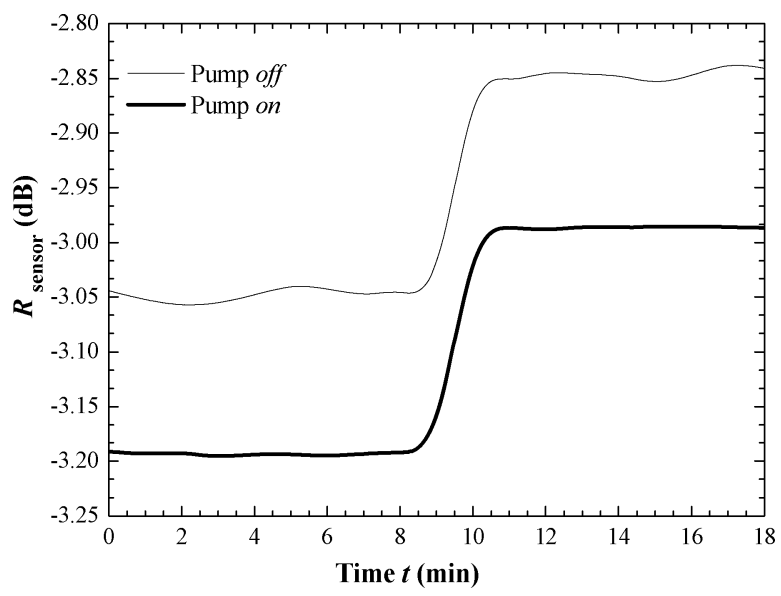

Fig. 5. Resolution evaluation of the optical fiber intensity sensor without and with amplification. (EDFA output power: $8 \mathrm{~mW}$.)

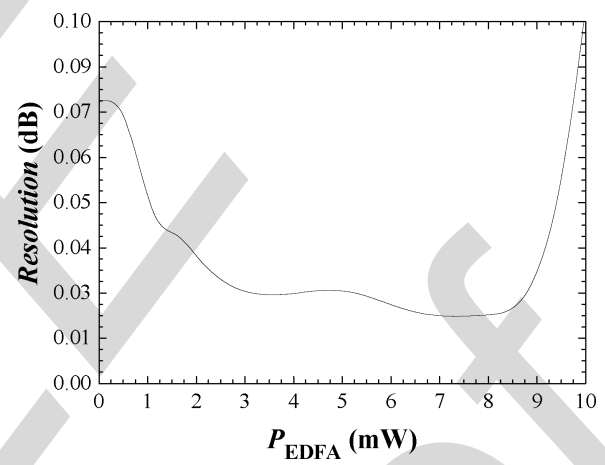

Fig. 6. Dependence of sensor resolution with the optical amplification level.

From the step changes and rms fluctuations observed in this figure, the resolution of the intensity sensor is calculated as $\approx 0.078 \mathrm{~dB}$ when the pump is off, becoming $\approx 0.025 \mathrm{~dB}$ when the pump is on, i.e., a factor of $\approx 3.1$ better. This result clear indicates the benefits of optical amplification in the sensor performance. Moreover, it is relevant to identify the dependence of the system resolution with the amplification level. This study is shown in Fig. 6, from where comes out the result that the optimum amplification is associated with a signal optical power in the range $\mathrm{mW}[3]-[8]$.

With larger amplification a fast degradation of the sensor resolution is apparent, a result of the increasingly important impact of the ASE noise in the detection system. Therefore, ASE can be a limiting factor in networks of optical fiber intensity sensors that need large levels of optical amplification. Anyway, it may be feasible to apply in this context results reported to some active bus network structures, where it has been demonstrated a nearly entire elimination of the ASE noise effects [15], [17], [18].

These values for the resolution were obtained from the sensor output signal that is given by (4), which is conditioned by the signal-to-noise ratios both in the signal and reference frequencies. Therefore, it is also interesting to study the dependence of these ratios with the pump power. When the pump power is off, an electrical signal-to-noise ratio of 15.5 and $13.8 \mathrm{~dB}$ for the reference and the sensor signal, respectively, were obtained. However, when the pump power of the EDFA was adjusted to 


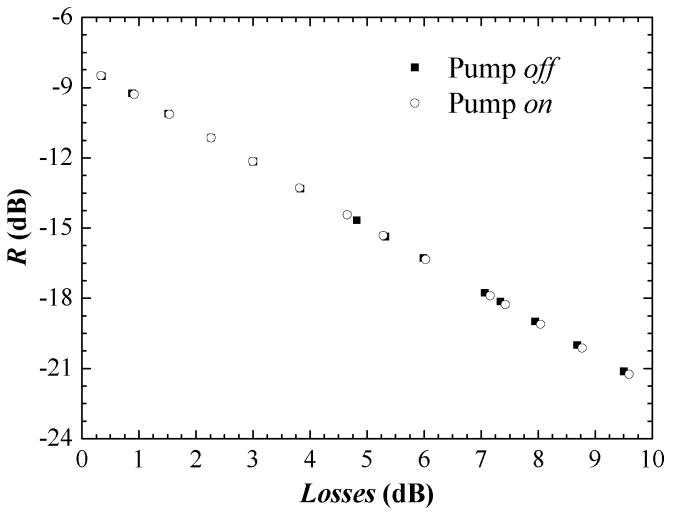

Fig. 7. Sensor characteristic function with and without optical amplification.

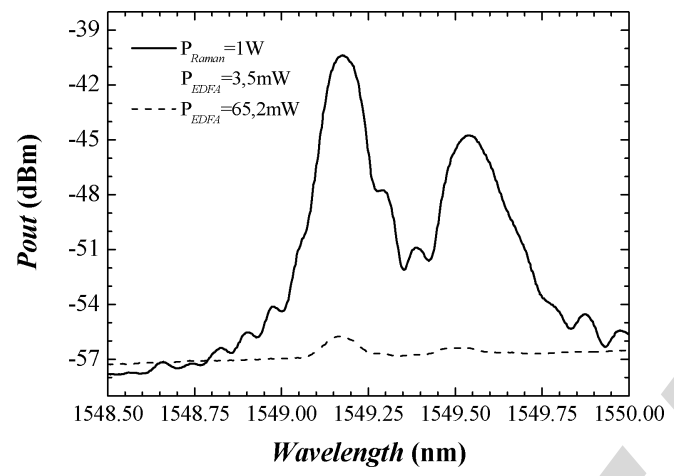

Fig. 8. Optical spectra for EDFA and hybrid Raman-EDFA amplification when $50 \mathrm{~km}$ of SMF was inserted into the setup.

provide a power of $8 \mathrm{~mW}$, these values improve to 24.7 and $25.05 \mathrm{~dB}$ for the reference and signal frequencies in that order. These better signal-to-noise ratios are then reflected in a better performance for the sensor output ( $R$-parameter). As a note, for intensity based sensors signal-to-noise ratios of the order of 20 $\mathrm{dB}$ are considered adequate for many applications [12].

To check if the sensor sensitivity is modified in the presence of optical amplification, the sensor characteristic function was plotted for the cases of optical amplification on and off. The results are shown in Fig. 7, from where it can be concluded that the sensitivity does not change when optical amplification is inserted into the system, as it should be considering the ratiometric processing employed [(4)]. The amplification improves the sensor performance because it induces better signal-to-noise ratios in the signal and reference frequencies.

To study the system behavior when the sensing head is located far away, $50 \mathrm{~km}$ of SMF were inserted in this setup. In that case, our signal has to go through $100 \mathrm{~km}$ of SMF, $50 \mathrm{~km}$ each way. First, an optical study of the reference and sensor response was done showing that the OSNR decreased too much by using only the EDFA for amplification. For that reason some variations were done in order to include also Raman amplification on it; such as the use of a WDM and an isolator to avoid reflections to the laser. As can be seen in Fig. 8, the OSNR obtained by using hybrid Raman-EDFA amplification was higher than using only EDFA.
Once again, computer simulations have been conducted to fine tuned all the parameters of the system. In this case, the frequency sweep applied to the carrier followed a sawtooth modulation $\left[f_{\text {mod }}=1 \mathrm{kHz}, \Delta f=21 \mathrm{kHz}, f_{0}=200 \mathrm{kHz}\right]$. Using these parameters our reference peak was located at 10 $\mathrm{kHz}$ and the signal peak at $12 \mathrm{kHz}$. For these parameters, the optimized inserted amplification into the setup was achieved using pumping powers of $1 \mathrm{~W}$ for the Raman laser and $3.4 \mathrm{~mW}$ for the EDFA. However, sensor resolution decreased substantially due to the noise inserted into the system by means of both amplifications (sensor resolution of about $0.915 \mathrm{~dB}$ ). Therefore, this result indicates the need to study in detail the effects that determine the system noise level in the presence of simultaneous Raman and EDFA amplification, looking for adequate performances in the context of interrogation of sensors located far away.

\section{CONCLUSION}

A frequency modulated continuous wave technique for referencing optical fiber intensity sensors using EDF amplification is reported. Without amplification, the system showed a sensor resolution of $\approx 0.078 \mathrm{~dB}$, a value that improves to $\approx$ $0.025 \mathrm{~dB}$ in the presence of a certain level of amplification. It was also identified an optimum optical amplification range and confirmed that the sensor sensitivity does not depend on the presence of optical amplification. We have also demonstrated that this configuration can be used as a remote sensing application. By using this technique, the sensor information can be read over a $50 \mathrm{~km}$ distance, although to achieve good performance careful optimization needs to be performed.

\section{REFERENCES}

[1] , J. M. López-Higuera, Ed., Handbook of Optical Fiber Sensing Technology. West-Sussex: Wiley, 2002.

[2] A. G. Stove, "Linear FMCW radar techniques," IEE Proc. F Radar and Signal Processing, , vol. 139, no. 5, pp. 343-350, Oct. 1992.

[3] D. Uttam and B. Culshaw, "Precision time domain reflectometry in optical fibre systems using a frequency modulated continuous wave ranging technique," J. Lightw. Technol., vol. 3, pp. 971-976, Oct. 1985.

[4] G. Zheng, M. Campbell, and P. A. Wallace, "Reflectometric frequencymodulation continuous-wave distributed fiber-optic stress sensor with forward coupled beams," Appl. Opt., vol. 35, no. 28, pp. 5722-5726, Oct. 1996.

[5] P. K. C. Chan, W. Jin, J. M. Gong, and M. S. Demokan, "Multiplexing of fiber Bragg grating sensors using an FMCW technique," IEEE Photonics Technol. Lett., vol. 11, pp. 1470-1472, Nov. 1999.

[6] P. A. Leilabady and M. Corke, "All-fiber-optic remote sensing of temperature employing interferometric techniques," Opt. Lett., vol. 12, no. 10, pp. 772-774, Oct. 1987.

[7] [Author: Please provide page range.] Saitoh, K. Nakamura, Y. Takahashi, H. Iida, Y. Iki, and K. Miyagi, "Ultra-long-distance (230 km) FBG sensor system," in Proc. 19th Int. Conf. Opt. Fiber Sensors, (OFS-19), 2008, vol. SPIE 7004, 70046C.

[8] Y.-G. Han, T. V. A. Tran, S.-H. Kim, and S. B. Lee, "Multiwavelength Raman-fiber-laser-based long-distance remote sensor for simultaneous measurement of strain and temperature," Opt. Lett., vol. 30, no. 11, pp. 1282-1284, Jun. 2005.

[9] Y.-G. Han, T. V. A. Tran, S.-H. Kim, and S. B. Lee, "Development of a multiwavelength Raman fiber laser based on phase-shifted fiber Bragg gratings for long-distance remote-sensing applications," Opt. Lett., vol. 30, no. 10, pp. 1114-1116, May 2005.

[10] J. H. Lee, Y. M. Chang, Y.-G. Han, H. Chung, S. H. Kim, and S. B. Lee, "Raman amplifier-based long-distance remote, strain and temperature sensing system using an erbium-doped fiber and a fiber Bragg grating," Opt. Express, vol. 12, no. 15, pp. 3515-3520, Jul. 2004. 
[11] Y.-J. Rao, Z.-L. Ran, and R.-R. Chen, "Long-distance fiber Bragg grating sensor system with a high optical signal-to-noise ratio based on a tunable fiber ring laser configuration," Opt. Lett., vol. 31, no. 18, pp. 2684-2686, Sep. 2006.

[12] S. Diaz, S. Abad, and M. López-Amo, "Fiber-optic sensor active networking with distributed erbium-doped fiber and Raman amplification," Laser Photon. Rev., vol. 2, no. 6, pp. 480-497, Dec. 2008.

[13] M. López-Amo, L. T. Blair, and P. Urquhart, "Wavelength-divisionmultiplexed distributed optical fiber amplifier bus network for data and sensors," Opt. Lett., vol. 18, no. 14, pp. 1159-1161, Jul. 1993.

[14] S. Diaz, B. Cerrolaza, G. Lasheras, and M. López-Amo, "Double Raman amplified bus networks for wavelength-division multiplexing of fiber optic sensors," J. Lightw. Technol., vol. 25, pp. 733-739, Mar. 2007.

[15] I. Labiano, S. Abad, and M. López-Amo, "Suppression of ASE effects in optically amplified sensor networks," in Proc. 16th Int. Conf. Opt. Fiber Sensors (OFS-16), 2003, pp. 364-367.

[16] V. Montoya, M. López-Amo, and S. Abad, "Improved double-fiber-bus with distributed optical amplification for wavelength-division multiplexing of photonic sensors," IEEE Photonics Technol. Lett., vol. 12, pp. 1270-1272, Sep. 2000.

[17] R. A. Perez-Herrera, O. Frazão, J. L. Santos, F. M. Araújo, L. A. Ferreira, J. M. Baptista, and M. Lopez-Amo, "Optical amplification to enhance a FMCW system for referencing intensity fibre optic sensors," in Proc. VI Symp. Enabling Opt. Netw. Sensors (SEON 2008), Porto, Portugal, 2008, pp. 67-68.

[18] C. Elosua, R. A. Perez-Herrera, M. Lopez-Amo, C. Bariain, R. GarciaOlcina, S. Sales, and J. Capmany, "Amplified CWDM self-referencing sensor network based on Phase-Shifted FBGs in transmissive configuration," in Proc. 19th Int. Conf. Opt. Fiber Sensors (OFS-19), Perth, Australia, 2008, pp. 700416-700416.

[19] P. K. C. Chan, W. Jin, and M. S. Demokan, "FMCW multiplexing of fibre Bragg grating sensors," IEEE J. Sel. Topics Quantum Electron., vol. 6, pp. 756-763, 2000.

[20] J. Zheng, "Analysis of optical frequency-modulated continuous-wave interference," Appl. Opt., vol. 43, no. 21, pp. 4189-4198, 2004.

[21] S. F. Collins, B. T. Meggitt, A. W. Palmer, and K. T. V. Grattan, "A multiplexing scheme for optical fibre interferometric sensors using an FMCW generated carrier," in Proc. 8th Int. Conf. Opt. Fiber Sensors (OFS-8), 1992, pp. 209-212.

[22] [Author: Please provide page range.] A. Perez-Herrera, D. A. Pereira, O. Frazão, J. M. C. Ferreira, J. L. Santos, F. M. Araújo, L. A. Ferreira, J. M. Baptista, and M. Lopez-Amo, "Frequency modulated continuous wave technique for referencing and multiplexing intensity based fiber optic sensors," in Proc. 19th Opt. Int. Conf. Opt. Fiber Sensors (OFS-19), 2008, vol. SPIE. 7004-206.

[23] A. D. Kersey, A. Dandridge, and K. L. Dorsey, "Transmissive serial interferometric fiber sensor array," J. Lightw. Technol., vol. 7, pp. 846-854, May 1989.

[24] A. D. Kersey, M. A. Davis, H. J. Patrick, M. LeBlanc, K. P. Koo, C. G. Askins, M. A. Putnam, and E. J. Friebele, "Fiber grating sensors," J. Lightw. Technol., vol. 15, pp. 1442-1463, Aug. 1997.

[25] P. Datta, I. Matías, C. Aramburu, A. Bakas, M. López-Amo, and J. M. Otón, "Tapered optical-fiber temperature sensor," Microw. Opt. Technol. Lett., vol. 11, no. 2, pp. 93-95, 1996.

[26] H. Masuda, "Review of wideband hybrid amplifiers," in Proc. Opt. Fiber Commun. Conf., 2000, vol. 1, pp. 2-4.

[27] F. J. Arregui, I. Matías, C. Bariain, and M. López-Amo, "Experimental design rules for implementation of biconically tapered singlemode fibre sensors," in Proc. I Eur. Workshop Opt. Fiber Sensors SPIE, Jul. 1998, vol. 3483, pp. 164-168.

[28] C. Bariain, I. R. Matías, F. J. Arregui, and M. López-Amo, "Experimental results toward development of humidity sensors by using a hygroscopic material on biconically tapered optical Fibre," in Proc. SPIE Opt. Fiber Opt. Sensor Syst., Sep. 1998, vol. 3555, pp. 95-105.

[29] F. J. Arregui, I. R. Matías, and M. López-Amo, "Optical fiber strain gauge based on a tapered single-mode fiber," Sens. Actuators A: Phys., vol. 79, no. 2, pp. 90-96, Jan. 2000.

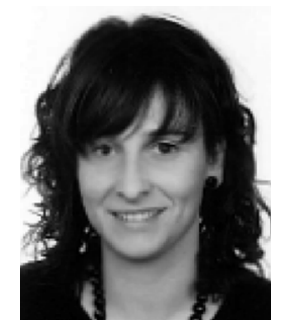

Rosa Ana Perez-Herrera was born in Cantabria, Spain, in February 1979. She received the Telecommunications Engineering degree from the University of Cantabria, Cantabria, Spain, in 2004. She is currently working towards the Ph.D. degree in the "Communications" Doctoral program at the Public University of Navarra, Pamplona, Spain.

In 2005, she obtained a scholarship from the Spanish Ministry of Science and Technology and joined the Optical Communications Group, Department of Electrical and Electronic Engineering, Public University of Navarra. During 2007, she was a visiting Ph.D. student at INESC at Unidade De Optoelectronica E Sistemas Electronicos, Porto, working on FMCW technique and in the summer of 2008 she carried out her joint research work in the School of Engineering and Mathematical Sciences, City University London, analyzing a range of multiwavelength ring laser configurations. In 2009, she became an Assistant Professor in the Electrical and Electronic Engineering Department, Public University of Navarra. Her research interests are in Raman amplifiers, erbium-doped amplifiers, fiber-optic sensors, and multiplexing architectures.

Orlando Frazão graduated with a Degree in physics engineering (optoelectronics and electronics) from the University of Aveiro, Aveiro, Portugal. He is currently working towards the Ph.D. degree in physics at the University of Porto, Porto, Portugal.

From 1997 to 1998, he was with the Institute of Telecommunications, Aveiro. Presently, he is a Researcher at Optoelectronics and Electronic Systems Unit, INESC Porto. He has published about 250 papers, mainly in international journals and conference proceedings, and his present research interests included optical fiber sensors and optical communications.

Mr. Frazão is a member of the Optical Society of America (OSA) and SPIE.

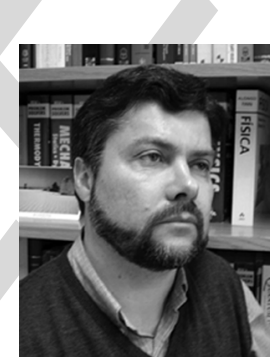

Jose Luis Santos graduated in 1983 with a Degree in applied physics (optics and electronics) from the University of Porto, Portugal, where he received the $\mathrm{Ph} . \mathrm{D}$. degree in physics in 1993 for research on fiberoptic sensing.

$\mathrm{He}$ is an Associate Professor in the Physics Department, University of Porto, and is in charge of the Optoelectronics and Electronic Systems Unit of INESC Porto. His main research interest is optical fiber sensing. He

Prof. Santos is a member of the Optical Society of America (OSA) and the International Society for Optical Engineers (SPIE) <<Author: Is SPIE correct $>>$.

Francisco M. Araújo graduated in 1993 with a Degree in applied physics (optics and electronics) from the University of Porto, Porto, Portugal, where he received the $\mathrm{Ph} . \mathrm{D}$. degree in physics in 2000.

$\mathrm{He}$ is a Senior Researcher with INESC Porto. His main activity involves optical communications and fiber-optic sensing. He is also a cofounder of FibreSensing, an INESC Porto spinoff company developing fiber-optic sensors and monitoring systems.

Luis A. Ferreira graduated with a Degree in applied physics optics and electronics in 1991 and received the M.Sc. degree in optoelectronics and lasers in 1995, the Ph.D. degree in physics in 2000 from the University of Porto, Porto, Portugal.

$\mathrm{He}$ is a Senior Researcher with INESC Porto. His main activity involves optical communications and fiber-optic sensing. He is also a cofounder of FibreSensing, an INESC Porto spinoff company developing fiber-optic sensors and monitoring systems. 


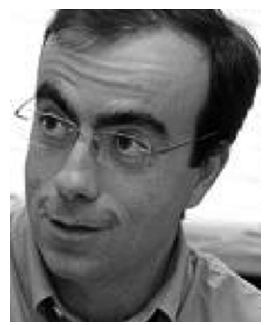

José M. Baptista (M’03) graduated with a Degree in electrical and computer engineering (telecommunications and computers) from the University of Porto, Porto, Portugal, in 1991, the M.Sc. degree in physics of laser communications from the University of Essex, Colchester, U.K., in 1992, and the Ph.D. degree in electrical and computer engineering from the University of Porto, in 2002.

Currently, he is an Assistant Professor at the University of Madeira and a Senior Researcher in the Optoelectronics and Electronics Systems Unit at INESC Porto. He is author of 37 journal publications and author of two patents. His research interests are in the area of fiber-optic sensors, fiber-optic communications, and fiber-optic technologies.

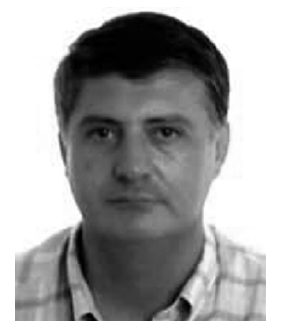

Manuel Lopez-Amo (M'91-SM'98) was born in Madrid, Spain, in 1960. He received the Degree in telecommunications engineering and the Ph.D degree from the Universidad Politécnica de Madrid, Spain in 1985 and 1989, respectively.

From 1985 to 1996, he belonged to the Photonic Technology Department, Universidad Politécnica de Madrid, where in 1990 he became an Associate Professor. During 1987, he was a visiting Ph.D student at INTEC of the University of Ghent, Belgium. In 1992, he was a Visiting Researcher at British Telecommunications Research Laboratories, Ipswich, U.K. Since 1996 he has been with the Public University of Navarra, Pamplona, Spain, where he became a Full Professor in the Department of Electrical and Electronic Engineering and is currently the Head of the Optical Communications Group. $\mathrm{He}$ has been Chairman of the Optoelectronic Committee of Spain. He has been leader of more than 20 research projects. He has coauthored more than 100 works in international refereed journals and conferences related with fiber-optic networks, optical amplifiers, fiber-optic sensors, and integrated optics.

Prof. Lopez-Amo is a member of the technical committees of the International Conference on Fiber Optic Sensors (OFS), the European Workshop on Optical Fiber Sensors (EWOFS), and the European Cost 299 Action, among others. He is a member of the Optical Society of America (OSA) and Electrical Optical Systems, Inc. (EOS). 